\title{
Politiques agricoles : libre-échange et nouvelles régulations
}

\author{
Jean-Paul JAMET \\ CNPA \\ 9 rue de la Baume, \\ 75008 Paris \\ <gisele.michelet@cnpa.asso.fr>
}

\begin{abstract}
The crisis that we have known, reflects structural imbalances that should not be underestimated. This article intends to draw on one hand, the contours of an enabling economic environment for development of world agriculture (rules for least developed countries, reducing risk and exposure of the agricultural economic fluctuations and market volatility ) and second, to review the challenges that agricultural development must now take into account, to be "sustainable" (new societal and environmental requirements).
\end{abstract}

Key words: agricultural economic fluctuations, least developped countries, reducing risk, environmental requirements
Les politiques agricoles n'échappent pas au contexte général de globalisation qui implique en corollaire, une plus grande ouverture des marchés. La conclusion de I'Uruguay Round à Marrakech en est la traduction, au point que I'Organisation mondiale du Commerce devient la principale institution de régulation des politiques agricoles. Le XXI ${ }^{\mathrm{e}}$ siècle verra par ailleurs s'accentuer la dépendance des zones déficitaires en productions agricoles (Asie du Sud-Est, zone méditerranéenne et moyen-orientale, au sens large notamment) vis-à-vis des zones appelées à être de plus en plus exportatrices (Amérique Latine, Bassin de la Mer Noire, Canada). Notons également l'évolution de plus en plus libérale de la Politique agricole commune qui participe au renforcement du dogme libre-échangiste.

Cette ambiance rend obsolète une bonne partie des politiques agricoles interventionnistes dans la gestion des marchés, même si elles sont relativement avantageuses en termes de dépenses publiques stricto sensu. Néanmoins, la récente crise agricole et alimentaire que nous venons de vivre doit être analysée, afin de définir de nouvelles modalités de régulation. Au-delà des évènements conjoncturels qui ont amplifié cette crise, quatre grandes explications méritent de retenir notre attention :

- un affaiblissement des politiques publiques de régulation des marchés dans les pays développés, initié lors de l'Uruguay Round et trouvant son aboutissement dans les réformes de la PAC, de 1991 à 2003 et une politique de libéralisation systématique de l'agriculture pratiquée par les grandes institutions internationales (FMI, Banque mondiale) vis-à-vis de pays en développement ;

- un rythme moins rapide de mise en valeur de nouvelles terres agricoles et de création de péri- mètres irrigués : par rapport à la période 19701990, le rythme de la mise en culture de nouvelles terres a été divisé par deux, sur la dernière décennie; de même les surfaces irriguées en Chine et dans la péninsule du Dekkan plafonnent, tandis que l'Amérique Latine et l'Afrique subsaharienne ne prennent pas le relais de cette extension de l'irrigation ;

- aucune politique volontariste et ample n'a été adoptée par les pays les plus pauvres, malgré l'engagement affiché par le Sommet mondial de l'alimentation, à Rome, de diminuer de moitié, entre 1992 et 2015, la faim et la malnutrition sur notre planète : au contraire, le nombre de personnes souffrant de faim est en train $d$ 'augmenter en valeur absolue, pour atteindre 923 millions de personnes, en 2007, selon la $\mathrm{FAO}$;

- un dérèglement monétaire et financier qui a amplifié les fluctuations des marchés de matières premières.

On peut considérer que la priorité depuis près de vingt-cing ans (Conférence inaugurale de Punta del Este, en 1985) a été donnée au développement des échanges de produits agricoles et alimentaires et que toute politique induisant une distorsion des échanges a été mise au banc des accusés et en demeure de se modifier. Les récentes crises agricoles et énergétiques ont ceci de commun, qu'elles traduisent un manque d'investissement, dans le système productif qui s'est ainsi montré plus vulnérable, à la fois à une réduction de l'offre et aux pressions de la demande. Même si les marchés se sont depuis retournés et à une grande vitesse, la crise que nous venons de connaître traduit des désajustements structurels qu'il convient de ne pas sous-estimer.

Mon ambition est de tracer, d'une part, les contours d'un cadre économique favorable au développement de l'agriculture mondiale et, d'autre part, de passer en revue les défis que le développement agricole doit dorénavant prendre en compte pour pouvoir être « soutenable » ou durable.

\section{Des règles d'exception pour les pays les moins avancés}

Comme l'a rappelé Marcel Mazoyer, le monde continuera de compter un grand nombre de ruraux et d'agriculteurs, notamment dans les pays les moins avancés d'Afrique, d'Amérique Latine et $d^{\prime}$ Asie. Dans ces pays, la productivité de l'agriculture est insuffisante pour que les agriculteurs puissent générer un cycle vertueux $\mathrm{d}^{\prime}$ amélioration de leurs moyens de production et de leur revenu. C'est une des grandes défaillances du Doha Round, prétendument appelé "Cycle du Développement », que de ne pas avoir prévu un cadre spécifique pour que les pays les moins avancés n'étant pas à même de faire décoller leur agriculture échappent aux contraintes de convergence des politiques de tarification et d'échanges énoncées par l'OMC. Lors du colloque organisé par Michel Barnier, le 3 juillet dernier, à Bruxelles, Pascal Lamy a justifié l'architecture globale de la négociation agricole à l'OMC qui prévoit des règles décalées pour les pays émergents (mise en œuvre plus longue et contrainte moins forte sur la baisse des tarifs et des soutiens). II a rappelé que la très grande majorité des pays en développement ne recourrait pas au plafond de protections douanières qui leur est permis et en a donc déduit que des règles d'exception pour les pays les moins avancées n'étaient pas nécessaires. 
Certes, les gouvernements des pays en difficulté de développement sont écartelés entre la satisfaction des besoins alimentaires des populations urbaines les plus pauvres (à partir d'importations à bon marché) et l'organisation d'une économie agricole grâce à des prix de vente rémunérateurs; mais est-il besoin de rappeler que la Chine et I'Inde viennent de démontrer que c'est I'amélioration de leur système agricole et alimentaire qui a permis leur décollage industriel.

Il faut que les grandes institutions internationales (OMC, Banque mondiale, FMI et FAO) affichent que la priorité pour un certain nombre de pays en voie de développement repose sur une démarche de développement autocentrée permettant la résolution de la question de I'auto-suffisance alimentaire. Ceci supposerait la création au sein de la FAO d'un Conseil de sécurité alimentaire, notamment chargé de l'évaluation du niveau de développement agricole et alimentaire des pays émergents. II s'agirait alors d'évaluer leurs performances en la matière et de définir des règles particulières de participation aux échanges internationaux de produits agricoles et alimentaires pour ces pays les moins avancés.

\section{Comment se protéger de l'instabilité et des risques?}

La deuxième disposition-cadre à réfléchir, concerne la question de la réduction du risque et de l'exposition des opérateurs économiques agricoles aux fluctuations et à la volatilité des marchés. Ce sujet demeure central.

Comme les travaux de Jean-Marc Boussard et Françoise Gérard sur les marchés agricoles I'ont explicité, dans le cas du modèle ID3 du CIRAD : "A la différence des modèles d'équilibre général, la production et la détermination des prix d'équilibre n'y sont pas simultanées. »

Les consommateurs sont approvisionnés par les récoltes de l'an dernier, tandis que les revenus sont distribués par les opérations de production de cette année, qui ne donneront de produits que l'an prochain. Les décisions de production sont donc prises sur la base de prix anticipés, et non de prix d'équilibre, comme dans le Cobweb d'Ezekiel.

En même temps, les producteurs savent que leurs anticipations ne se réaliseront pas forcément. Ceci les conduit à prendre des primes de risque et à calculer leurs coûts de production en conséquence. Ce modèle ne prend pas en considération les accidents climatiques, ni la croissance des agrocarburants, ni le comportement des « spéculateurs ». Pourtant, en se plaçant dans la situation de 2001, il projette dans le long terme une hausse des prix du même ordre que celle observée durant la campagne $2007 / 2008$. Cela permet de penser que le rôle des anticipations et du risque dans le comportement des agriculteurs est correct.

La dérégulation des marchés agricoles ne met pas à l'abri des crises et ne réduit pas forcément I'instabilité des prix. L'incertitude sur les prix entraîne des effets souvent asymétriques: le consommateur est plus rapidement touché par la hausse et alors qu'il attend les effets des baisses qui sont souvent atténuées. Par ailleurs, l'incertitude sur les prix inquiète le producteur et freine les investissements et le progrès technique de manière très significative.

L'ouverture des marchés agricoles rend obsolète la plupart des politiques classiques d'intervention sur les marchés. Pourtant, un défi demeure pour les politiques publiques: celui de la réduction des risques, dans un contexte d'imperfection du marché.

Dans le modèle théorique de concurrence parfaite de Pareto, le jeu de marché conduit à une allocation optimale des ressources. Mais le monde n'est pas parfait et la concurrence ne conduit donc pas à une allocation de premier rang des ressources.

Les origines de ces défaillances du marché sont multiples et beaucoup de travaux récents d'économie reposent pour l'essentiel sur l'existence d'asymétries d'information.

\section{Améliorer la qualité de l'information sur les composants classiques des marchés agricoles}

Il appartient désormais aux institutions publiques de produire une information de meilleure qualité. II s'agit d'abord d'améliorer la qualité de l'information sur les composants classiques des marchés agricoles : état de l'offre des différentes productions, état des demandes relatives aux différentes utilisations des produits agricoles et forestiers, état des stocks de report relativement au déroulement des différentes campagnes.

Cette information doit se caractériser par une disponibilité maximale et une actualisation fréquente. Par sa fiabilité et sa transparence, elle doit permettre à tous les acteurs de prendre les décisions de production les plus pertinentes, pour satisfaire les exigences des différents utilisateurs de produits agricoles.

II existe un exemple de réalisation assez probant de cette démarche, dans le cas de l'Agence internationale de l'énergie qui éclaire, par son travail d'expertise et de synthèse, la situation des marchés des différentes commodités énergétiques. Mais, même dans ce cas, M. Mandil, son ancien directeur, a émis des recommandations aux autorités européennes pour qu'elles complètent, par une démarche semblable, la production de données statisti- ques hebdomadaires sur les marchés européens, afin que ce ne soient pas les seules informations hebdomadaires sur l'état des stocks de produits pétroliers aux États-Unis qui servent de références aux opérateurs du marché.

Ainsi, si un Observatoire mondial de l'alimentation, à la structure relativement légère et réactive, devait être créé dans les secteurs agricoles, forestiers, alimentaires et non alimentaires, il conviendrait de l'accompagner par des initiatives régionales, afin de disposer d'une information la plus détaillée possible.

On peut aisément imaginer que l'analyse des informations statistiques puisse aboutir, au niveau du Conseil de sécurité alimentaire évoqué plus haut, à faire des recommandations sur la situation des stocks des différents produits et sur les arbitrages à opérer entre les différentes utilisations, qu'il s'agisse, par exemple, de l'aide alimentaire ou de la production de certains agrocarburants qui pourrait être plafonnée, dans le cas de stocks de reports insuffisants. On peut penser aussi aux récentes tensions sur le marché du riz qui eussent été beaucoup moins fortes, si les grands exportateurs comme le Vietnam ou l'Inde n'avaient pas interdit les exportations sur le marché international.

\section{Une politique dynamique de gestion des stocks agricoles}

Il convient d'attacher une attention particulière à la politique de gestion des stocks. Celle-ci peut continuer d'avoir une certaine légitimité, dans un marché ouvert avec une finalité légèrement différente que celle $d^{\prime}$ un filet $d$ 'intervention : le but de la constitution de stocks de sécurité alimentaire ne serait pas l'action directe sur le niveau de prix, mais plutôt le rôle d'amortisseur sur la volatilité des marchés. En effet, un excès de volatilité ne peut que conduire au désordre et, dans le cas de nombreux marchés agricoles, à l'amplification de cycles de production qui, finalement, ne sont bénéfiques ni pour les producteurs ni même pour les consommateurs. Ces cycles constituent par ailleurs une difficulté supplémentaire pour les agriculteurs des pays où l'agriculture est la moins organisée.

"La propension des Gouvernements à instaurer des embargos à l'exportation serait beaucoup moins forte, s'ils acceptaient de détenir des stocks de réserve mobilisables, en cas de chute de production ou d'une augmentation soudaine de la demande » rappelle Daryll Ray, professeur à l'université du Tennessee. Or, la détention de stocks publics à des fins de sécurité alimentaire est une mesure admissible dans la boîte verte de l'Accord de Marrakech. 


\section{La gestion des risques : des politiques divergentes entre l'Europe et les États-Unis}

La gestion des risques conduit à des politiques agricoles contrastées de chaque côté de l'Atlantique: du côté de I'Union européenne, la Politique agricole commune cherche à se mouler le plus possible dans les contraintes négociées à l'Organisation mondiale du Commerce.

\section{Union européenne : un manque d'ambition très réel}

Pour la Commission européenne, le découplage des aides directes doit conduire à un lissage du soutien du revenu des agriculteurs et ne nécessite donc pas de dispositif anti-crise particulier, hormis les calamités climatiques exceptionnelles et les crises sanitaires systémiques.

La Commission européenne ne veut plus interférer dans l'orientation des productions agricoles, sauf pour des raisons de conditionnalité des aides (environnement, bien-être animal et sécurité sanitaire des aliments) ou de développement territorial, agricole et rural, dans le cas des zones fragiles et des zones de montagne. L'incitation à une gestion des risques économiques par un système $d^{\prime}$ assurance où la responsabilité serait partagée entre les agriculteurs, les organismes de solidarité mutualiste et les pouvoirs publics, qu'ils soient nationaux ou communautaires, n'est envisagée que pour des programmes d'assurance-récolte, en cas de catastrophe naturelle et fonds de mutualisation en cas de maladies animales dans les nouveaux articles 68,69 et 70 proposés dans le bilan de santé 2008 de la PAC.

Le nouveau règlement rappelle que " compte tenu de l'importance croissante d'une gestion efficace des risques, il importe de donner aux Étatsmembres la possibilité de prendre en charge une partie des primes d'assurance-récolte payées par les agriculteurs ou de compenser financièrement certaines pertes économiques liées à des maladies animales ou végétales. Pour que la Communauté puisse respecter ses obligations internationales, les ressources pouvant être affectées à des mesures de soutien couplé doivent être limitées à un niveau approprié ».

La Commission fait donc un premier pas, mais l'ampleur que pourrait prendre cette politique après 2013 dépend beaucoup du nombre d'États membres qui utiliseront la possibilité qui leur est offerte. Le manque d'ambition de la Commission dans ce domaine s'explique par les trois raisons suivantes:

- I'hétérogénéité de ce traitement assurantiel, dans les vingt-sept États membres de I'Union qui rend difficile la définition d'une politique commune harmonisée dans ce domaine. La démarche de simplification de la PAC pratiquée par Mme Fischer-Boël est incompatible avec un exercice conduisant à concevoir autant de dentelles que d'Etats membres ;

- la possibilité de proposer des dispositifs équilibrés entre productions, conduirait à des injustices pour les productions les moins prises en charge aujourd'hui : on pense, en particulier, au cas des productions animales où les facteurs de risques sont multiples ;

- une évaluation du risque très inégal, selon les régions et les structures d'exploitation.

Cette sorte de capitulation est pourtant inacceptable. L'imperfection des services de gestion des risques offerts aux agriculteurs justifie l'engagement des pouvoirs publics, dans ce domaine. L'intervention publique doit être associée à des dispositifs privés, la difficulté étant de définir les modalités optimales de cette combinaison public/privé. C'est pourquoi on pourrait imaginer la création d'une agence européenne qui accompagnerait la mise en place des politiques assurantielles et disposerait également d'une capacité financière de lissage indispensable à la pérennisation d'une telle politique au plan communautaire (compte tenu des contraintes de l'annualité budgétaire).

\section{Amérique du Nord : une panoplie d'instruments}

Comparativement, les processus mis en place en Amérique du Nord, qu'il s'agisse du Canada ou des États-Unis, font la part belle à toute une panoplie d'instruments permettant la gestion des risques. Les politiques publiques cumulent en effet des dispositifs complémentaires :

- les aides directes ;

- les soutiens aux dispositifs d'assurancerécolte et chiffre d'affaires où les pouvoirs publics assurent une grande part de responsabilité ;

- le soutien significatif aux agrocarburants.

Les paiements directs aux agriculteurs américains peuvent varier énormément d'une campagne à I'autre (24,4 milliards de dollars, en 2005 contre par exemple 13,6 milliards en 2007).

Ils comprennent :

- les aides fixes découplées créées par le Fair Act de 1996, comprises selon les années entre 5 et 5,3 milliards de dollars :

- les aides anticycliques basées sur la différence entre le prix de marché et le prix d'objectif et rapportées à la surface de l'exploitation. Ces paiements se sont élevés à 4,1 milliards, en 2005 et 2006, mais seulement à 1 milliard, en 2007 ;
- les marketing loans sont passés, pour leur part, de 5,5 milliards de dollars en 2005 à 0,1 milliard en 2007.

Le coût du programme de jachère environnementale est en progression (2,3 milliards de dollars en 2004 contre 3,1 milliards de dollars, en 2007).

Les aides aux calamités agricoles ont été très variables: 0,6 milliard de dollars en 2004, 3,2 milliards de dollars en 2005, 0,3 milliard en 2006 et 2 milliards en 2007.

À cela, il faut ajouter les aides d'urgence et différentes subventions comprises entre 3,7 milliards en 2005 et 2 milliards en 2007.

La nouvelle loi-cadre agricole dénommée FCEA (Food Conservation and Energy Act of 2008) propose une option par rapport aux paiements anticycliques, au travers du programme ACRE (Average Crop Revenue Election).

Ce programme vise à stabiliser les recettes des producteurs de grandes cultures, sur une base pluriannuelle. Le paiement reçu par le producteur est basé sur la différence du chiffre d'affaires (prix $\times$ rendement $d^{\prime}$ un producteur et $d^{\prime}$ un chiffre d'affaires national). Le chiffre d'affaires historique par culture correspond à $90 \%$ du rendement quinquennal de chaque État et du prix moyen du marché enregistré au niveau national, pendant les deux campagnes précédentes.

Pour participer à ACRE, un producteur doit renoncer complètement aux paiements anticycliques et accepter une réduction de $30 \%$ du prix minimum et de $20 \%$ du paiement fixe par culture. S'il souscrit à ce programme, disponible à partir de la récolte 2009, ce sera pour toutes les cultures éligibles de l'exploitation et pour toutes les campagnes suivantes jusqu'en 2012.

Ce programme est basé sur l'observation que les rendements et les prix des cultures varient de façon inverse, et donc, qu'il devrait être plus avantageux pour la puissance publique de s'intéresser au chiffre d'affaires qui tamponne ces fluctuations. On se rapproche ainsi de la démarche assurantielle qui bénéficie d'incitations significatives du gouvernement fédéral.

Les aides aux assurances se sont élevées à 3,8 milliards de dollars en 2007 et pourraient atteindre presque 6 milliards de dollars en 2008. Ces derniers se décomposent en 1,3 milliard d'aide forfaitaire aux compagnies $d^{\prime}$ assurance pour les dédouaner des frais de souscription de contrat. Quatre milliards correspondent à la prise en charge du coût de la police par l'agriculteur, en moyenne $60 \%$ du coût de la police, mais ce taux est variable selon le niveau de couverture des risques (le taux de prise en charge peut même atteindre $70 \%$ ). Enfin, l'État assure aussi la garantie de bonne fin et joue le rôle de réassureur. 
Le niveau des garanties est passé de 30 milliards de dollars en 1999 à 66 milliards de dollars, en 2007. Il y a une dizaine d'années, les contrats portaient surtout sur les rendements. Aujourd'hui, ils portent essentiellement sur le chiffre d'affaires.

N'oublions pas de mentionner, à propos de la politique agricole américaine, deux autres domaines $d$ 'intervention significatifs: les aides alimentaires et la politique de soutien aux agrocarburants.

On peut estimer à 7 milliards de dollars en 2008, le coût du soutien fédéral, principalement à l'éthanol de maïs. C'est ainsi que le mélangeur qui respecte le taux $d^{\prime}$ incorporation obligatoire, bénéficie d'un crédit d'impôt de 51 cents par gallon. Le marché de l'éthanol est par ailleurs protégé par un droit de douane de 54 cents par gallon.

L'aide alimentaire se répartit en trois programmes principaux : bons alimentaires $(38,5$ milliards de dollars en 2008), les cantines scolaires (14,7 milliards de dollars) et l'aide pour les femmes, enfants et nouveau-nés (6,2 milliards de dollars).

\section{De nouvelles exigences sociétales et environnementales}

Les progrès de productivité de l'agriculture entraînent des revers que la société veut voir corrigés, qu'il s'agisse de :

- la consommation des engrais qui peut conduire à des rejets trop importants de nitrates dans les nappes phréatiques, les cours d'eau et les rivages;

- la maîtrise de I'utilisation des herbicides et des pesticides dont la stabilité des molécules peut entraîner des nuisances mal connues et générer une pollution des fonds ;

- la maîtrise des effluents d'élevage avec leur charge en nitrates et phosphates, au regard de la qualité des eaux ;

- la gestion équilibrée des sols, grâce au maintien de la teneur en humus (en réinventant une complémentarité agriculture-élevage), de la lutte contre l'érosion et du maintien d'un couvert végétal ;

- la contribution à la restauration de la biodiversité, à la fois grâce à la diversification des cultures et à une meilleure organisation territoriale organisant la complémentarité entre agriculture, haies, taillis et forêts.

\section{La pertinence d'une intégration territoriale}

L'Union européenne a pris en compte ces attentes sociétales au travers du principe de la conditionnalité des aides (pénalisation possible des DPU) et dans la mise en œuvre de la politique de développement rural mobilisant à la fois des moyens communautaires, nationaux et régionaux.

Il est manifeste que si l'on veut aller plus loin dans la prise en compte des contraintes environnementales, au sens large (incluant le changement climatique, le maintien de la biodiversité et le bien-être animal), la modulation et l'intégration territoriale de ces différentes approches en conditionnent l'efficacité.

Je partage, à cet égard, I'analyse de Gilles Lemaire: "La plupart des problèmes environnementaux auxquels l'agriculture est confrontée (...) ne peut s'appréhender et $n^{\prime} a$ de réelle pertinence qu'à des échelles beaucoup plus larges que celles des territoires des exploitations agricoles: bassin versant, impluvium de nappe, petite région agricole, voire les continents et la planète toute entière (...). L'évolution récente de l'agriculture "s'est traduite par une simplification des assolements et des rotations engendrant dans ces régions, des atteintes sévères à l'environnement, notamment au travers de la qualité des eaux. Dans ces régions, cette intensification et la simplification poussée à l'extrême de la mosaïque paysagère (diminution de la diversité des cultures et augmentation de la taille des parcelles) a engendré une diminution de la biodiversité, tant au niveau des plantes que des insectes, des micromammifères et des oiseaux (...). La disparition des surfaces pérennes dans les grandes plaines céréalières s'est accompagnée par une érosion importante de la biodiversité... C'est le maintien sur un même territoire, d'une diversité de systèmes de production complémentaires qui engendre une diversification des modes d'occupation des sols, des assolements et des rotations et permet d'optimiser des flux de carbone et d'azote, à l'échelle locale, afin de mieux "boucler" les cycles biogéochimiques. Le maintien de la diversité des systèmes de production est la solution pour concilier les fonctions de productivité et les fonctions environnementales de l'agriculture. »

Les modalités de rémunération des externalités agricoles, au regard des services environnementaux qu'elles sont susceptibles de rendre, méritent une analyse particulière.

Comme le soulignait Dominique Bureau, dans un rapport au Conseil d'analyse économique, «il faut souligner que l'approche contractuelle globale a souvent été envisagée pour rémunérer les avantages territoriaux. Elle apparaît séduisante par la possibilité qu'elle offre, de s'adapter aux terroirs et d'incorporer au contrat, de multiples dimensions ». Mais elle s'accompagne de coûts de transaction très élevés, d'où l'idée de " recommander des subventions ciblées versées par des Agences de bassin, à l'image de ce qui se fait pour les eaux, mais on pourrait en créer aussi des équivalentes pour la biodiversité ".

Il faut donc, dans cette optique, envisager l'incitation à des politiques contractuelles de moyen et long terme entre des autorités décentralisées et chaque exploitation agricole. Ce sera sans doute la manifestation et l'explicitation d'un nouveau contrat social entre les agriculteurs, les éleveurs, les forestiers et le reste de la société.

\section{Hiérarchisation et cohérence au niveau planétaire}

Mais les défis de demain sont aussi globaux, qu'il s'agisse du changement climatique, du maintien des forêts, notamment équatoriales, de la maîtrise des émissions de gaz à effet de serre, du développement agricole des pays les moins avancés et de l'équilibre à penser entre usages alimentaires, combustibles, carburants et chimie verte.

Plus que jamais, une gouvernance mondiale, pour définir une vision globale sur la production biologique de la planète, dans les domaines agricoles, forestiers et marins, s'impose afin d'identifier les priorités, les complémentarités et les incompatibilités entre filières.

Cette mobilisation, au niveau planétaire ne prendra pas facilement corps, mais on peut souhaiter une structuration autour de deux axes :

- La dynamisation du rôle de la FAO, par la création en son sein, d'un Conseil de sécurité alimentaire fait d'une vingtaine de pays, afin de pouvoir prendre des résolutions rapidement et de participer à la hiérarchisation des grandes politiques de régulation qui se mettent en place : harmonisation des échanges (OMC), priorité au développement agricole pour les pays les moins avancés (Cnuced), maîtrise de la déforestation, maîtrise du changement climatique (GIEC et protocole de Kyoto), etc.

- Ce rôle de pilotage sera d'autant plus efficace qu'un effort sera fait pour rendre plus réactive l'information économique sur les marchés agricoles qui fait déjà l'objet d'un travail de la FAO et de l'OCDE.

Enfin, ces efforts doivent être menés parallèlement dans chaque grande région de notre planète. 